\title{
A ROBUST CALCULUS OFFLOADING STRUCTURE FOR AMBULANT HEALTH INQUIRIES
}

\author{
Pitta Rebecca Alekhya ${ }^{1}$, K. Tulasi Krishna Kumar Nainar ${ }^{2}$ \\ ${ }^{\text {I}}$ Student-CSE Department, Sanketika Vidya Parishad Engineering College, Visakhapatnam, \\ Andhra Pradesh. \\ ${ }^{2}$ Associate Professor-CSE Department, Sanketika Vidya Parishad Engineering College, \\ Visakhapatnam, AP
}

Article DOI: https://doi.org/10.36713/epra8437 DOI No: 10.36713/epra8437

\begin{abstract}
Recently, research intergrading medicine and Artificial Intelligence has attracted extensive attention. Mobile health has emerged as a promising paradigm for improving people's work and life in the future. However, high mobility of mobile devices and limited resources pose challenges for users to deal with the applications in mobile health that require large amount of computational resources. In this paper, a novel computation offloading mechanism is proposed in the environments combining of the Internet of Vehicles and Multi-Access Edge Computing. Through the proposed mechanism, mobile health applications are divided into several parts and can be offloaded to appropriate nearby vehicles while meeting the requirements of application completion time, energy consumption, and resource utilization. A particle swarm optimization based approach is proposed to optimize the aforementioned computation offloading problem in a specific medical application. Evaluations of the proposed algorithms against local computing method serves as base line method are conducted via extensive simulations. The average task completion time saved by our proposed task allocation scheme increases continually compared with the local solution. Specially, the global resource utilization rate increased from $71.8 \%$ to $94.5 \%$ compared with the local execution time.
\end{abstract}

KEY WORDS: Computation Offloading, Mobile Health, Internet of Vehicles, Multi-Access Edge Computing.

\section{INTRODUCTION}

ARTIFICIAL Intelligence (AI) and big-data technology have developed continuously in recent years, garnering great scholarly interest. Healthcare is an important AI application area which is limited by the performance of computing devices given the enormous amount of computation required by such devices. Computer-aided diagnosis (CAD) is a particularly promising domain. CAD is mainly about assisting clinical diagnosis by computer technology accurately. Recently, cloud computing (CC) technology, which can share computing resources throughout a network, has become a supporting technology for $\mathrm{AI}$; $\mathrm{CC}$ has enabled $\mathrm{AI}$ to be incorporated into various fields, of which medical applications are especially popular. With the increasing demand for medical services, mobile health applications have been studied extensively. Through which, a new kind of healthcare application combining medical applications with devices is generated.

\section{OBJECTIVE OF THE PROJECT}

This algorithm has attracted academic attention owing to its advantages of simple implementation, high precision, and fast convergence. It is also demonstrably superior in solving practical problems. With the advantages of edge computing and $\mathrm{V} 2 \mathrm{~V}$ communication technologies, rescue organization can efficiently solve computation offloading problems for mobile health applications. We propose a novel computation 


\section{EPRA International Journal of Research and Development (IJRD)}

offloading mechanism by formulating a non-linear integer programming problem, where each application is divided into several parts and offloaded to nearby vehicles.

\section{PURPOSE OF THE PROJECT}

Proposed a forward central dynamic and available approach by adapting the running time of sensing and transmission processes in IoT-based portable devices, a system-level battery model by evaluating the energy dissipation in IoT devices and a data reliability model for edge artificial intelligence based IoT devices over hybrid TPC and duty-cycle network. And proposed two novel algorithms named, Power-aware QoE Optimization and Buffer-aware QoE Optimization, to improve lifetime of portable devices. It can be seen from the above that the energy limitation carried by portable devices is a problem that researchers want to overcome.

\section{LITERATURE REVIEW}

"A new optimizer using particle swarm theory," The optimization of nonlinear functions using particle swarm methodology is described. Implementations of two paradigms are discussed and compared, including a recently developed locally oriented paradigm. Benchmark testing of both paradigms is described, and applications, including neural network training and robot task learning, are proposed. Relationships between particle swarm optimization and both artificial life and evolutionary computation are reviewed.

\section{"Evolution of 5G in Internet of Medical Things,"}

3D ultra-high resolution videos can be downloaded within seconds by deploying state-of-the art 5G technology. It handles big data with lesser delay and provides more bandwidth. The Internet of things (IoT) is the physical networks which different technologies together Wi-Fi, Bluetooth and Cellular on a one platform. The IoT for medical healthcare which is IoMT requires high data, high speed and long battery life with reliable connectivity. The 5G fulfills these requirements with its superfast capabilities and supports IoMT for human health such as, diagnosis and treatment. This paper presents the detailed survey about how 5G has revolutionized the medical healthcare with the help of IoT for enhancing quality and efficiency of the wearable devices. Besides, state-of-the art 5G-based sensor node architecture is proposed for the health monitoring of the patients with ease and comfort.

"Medical-QoS based telemedicine service selection using analytic hierarchy process,"

An emerging breakthrough paradigm shift in health industry and wearable devices, large scale and distributed mobile cloud computing technologies have led to new opportunities for medical healthcare systems. Telemedicine service selection and management of Medical-Quality of Service (m-QoS) in large-scale and distributed medical health system (e.g. medical data centers, hospitals, medical servers and medical clouds, etc.) is a key challenge for both industry and academia. The aim of this chapter is to improve and manage $\mathrm{m}-\mathrm{QoS}$ by prioritizing Telemedicine service by using decisive and intelligent tool called Analytic Hierarchy Process (AHP). This service will be provided on urgency basis from the pool of medical services with the help of AHP. In this connection, four telemedicine services are considered i.e. Tele-surgery, Tele Consultation, Tele-Education and Tele-Monitoring. In this research, three m-QoS parameters are considered i.e. throughput, jitter and delay. These services are evaluated by potential doctors and patients. We propose an AHP based decision making algorithm for selecting urgent and important service for the fast and cost-effective treatment of the emergency patients at the remote location in the hospital, because AHP is the significantly fast decision making technique used to assess, select and manage the emergency services at various priority levels in large scale and distributed medical health systems.

\begin{abstract}
"Mobile e-health: The unwired evolution of telemedicine,"

The movement of telemedicine from desktop platforms to wireless and mobile configurations may have a significant impact on future health care. This paper reviews some of the latest technologies in wireless communication and their application in health care. The new technologies can make the remote medical monitoring, consulting, and health care more flexible and convenient. But, there are challenges for successful wireless telemedicine, which are addressed in this paper.
\end{abstract}

"A survey on M2M systems for $m$ Health: A wireless communications perspective,"

In the new era of connectivity, marked by the explosive number of wireless electronic devices and the need for smart and pervasive applications, Machine-to-Machine (M2M) communications are an emerging technology that enables the seamless device interconnection without the need of human interaction. The use of M2M technology can bring to life a wide range of $\mathrm{m}$ Health applications, with considerable benefits for both patients and healthcare providers. Many technological challenges have to be met, however, to ensure the widespread adoption of $\mathrm{m}$ Health solutions in the future. In this context, we aim to provide a comprehensive survey on M2M systems for $\mathrm{m}$ Health applications from a wireless 


\section{EPRA International Journal of Research and Development (IJRD)}

communication perspective. An end-to-end holistic approach is adopted, focusing on different communication aspects of the M2M architecture. Hence, we first provide a systematic review of Wireless Body Area Networks (WBANs), which constitute the enabling technology at the patient's side, and then discuss end-to-end solutions that involve the design and implementation of practical $\mathrm{m}$ Health applications.

\section{GENETIC ALGORITHM}

This algorithm has attracted academic attention owing to its advantages of simple implementation, high precision, and fast convergence. It is also demonstrably superior in solving practical problems. PSO, developed by $\mathrm{J}$.
Kennedy and R.C. Eberhart, is an optimization method inspired from the ability of social organizations to face and solve complex problems involving the individual capacities of their members and also the group social dynamics. In PSO, a particle is informed about its neighborhood and also about the group. The best neighborhood solution as well as the best global solution is used by the particle to adjust its forthcoming displacement. It begins with a random solution and finds the optimal solution through iteration, evaluated on the basis of fitness. PSO is simpler than the genetic algorithm and does not include the cross- and mutation operations of the genetic algorithm; instead, PSO finds the global optimal solution by following the currently searched optimal value.

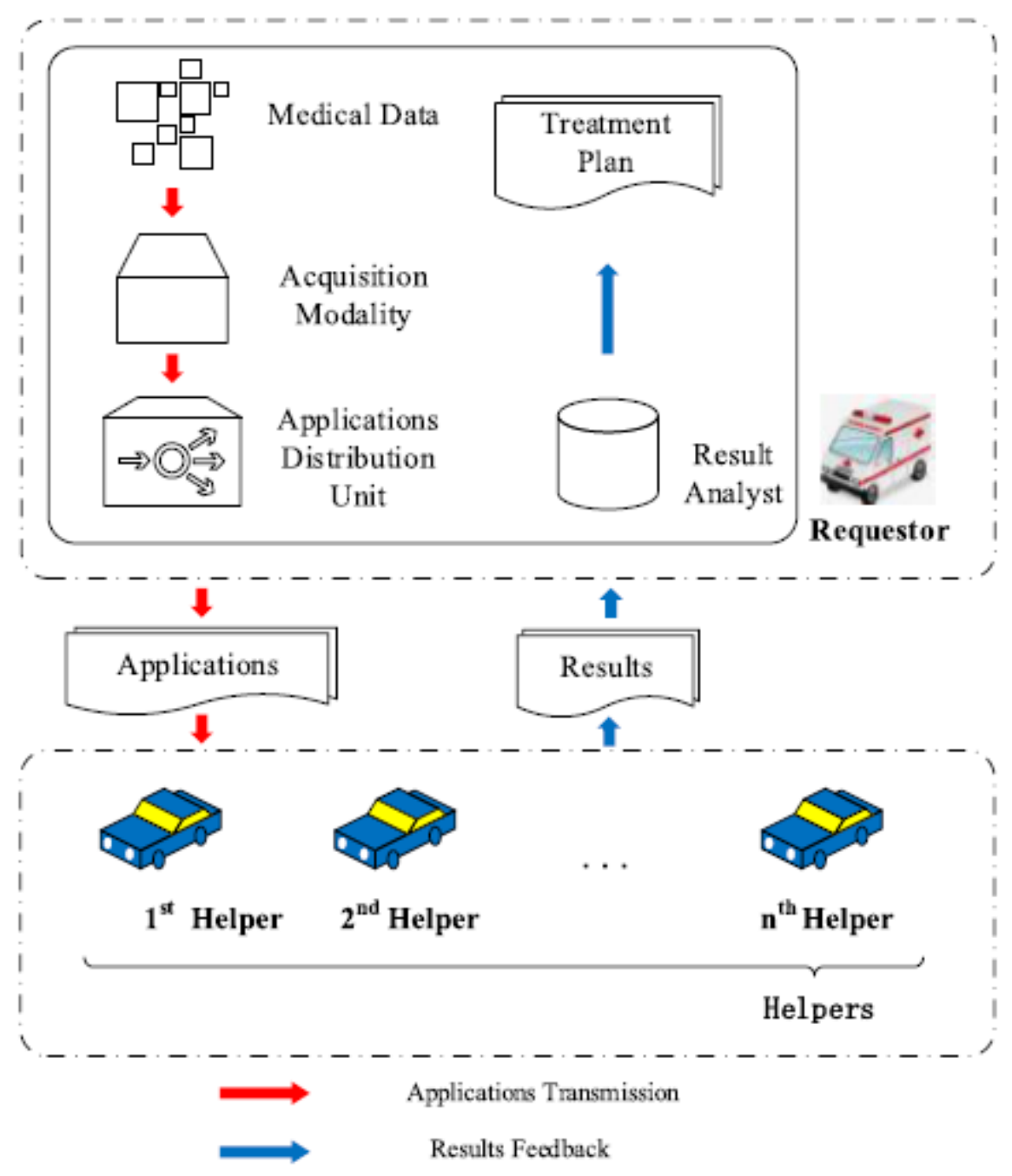

Fig 1. System Architecture 


\section{EPRA International Journal of Research and Development (IJRD)}

\section{MODULES}

\section{Computer aided diagnosis}

Computer-aided diagnosis (CAD) is a particularly promising domain. CAD is mainly about assisting clinical diagnosis by computer technology accurately. Recently, cloud computing (CC) technology, which can share computing resources throughout a network, has become a supporting technology for AI; CC has enabled AI to be incorporated into various fields, of which medical applications are especially popular. With the increasing demand for medical services, mobile health applications have been studied extensively. Through which, a new kind of healthcare application combining medical applications with devices is generated.

\section{Internet of vehicles}

The former is mainly used for health monitoring in people's daily lives, whereas the later is primarily intended for telemedicine in remote regions and medical rescue in disaster-stricken areas. This paper primarily addresses medical rescue in disaster where communication and power resources are constantly cut off. A temporary self-organizing network of vehicles can be established through vehicle-tovehicle (V2V) technologies to meet the communication needs of medical rescuers. In both above mentioned forms, AI technology is utilized as an emergency replacement in cases where manual diagnosis cannot be obtained expediently which also plays an important part in mobile health care. Such applications have serious demands on computational resources and powerful capabilities.

\section{Multi-Access Edge Computing}

According to which, users and the cloud cannot maintain a stable connection. The resource capacity of CC therefore can hardly meet the demand of mobile health applications. Therefore, the MultiAccess Edge Computing (MEC) is introduced along with the 5th generation (5G) communication technology and the Internet of Vehicles (IoV).
Comparing with CC, MEC can provide wireless users with services that alleviate non-ideal transmission delays and severe degradation. However, mobile edge servers may still experience signal coverage limitations and resource constraints in cases of high user density, especially during high-traffic periods.

\section{Application Distribution Model}

In this paper, typical mobile health applications are mainly considered in disaster conditions (e.g., a refugee rescued from ruins during an earthquake who is in critical condition). Emergency CT equipment and clinical examination equipment, such as for blood testing, can quickly gather the required patient information via Mobile health applications. Each application has several information forms (e.g., an electrocardiograph report with corresponding data [time series], blood test report [key value pairs], and CT scans [images]), which are inherently complicated and difficult to process without enough computation resource. In a scenario with limited computational resources, satisfactory application completion time requirements cannot be met when running.

\section{Running Time Performance}

Running time performance is a key concern in mobile health scenarios involving disasters. Therefore, we first compare the running time performance of the proposed mechanism with the optimal results, as shown. To generalize the discussion to various traffic conditions, we evaluated 1000 times simulations through Monte Carlo method per group under different traffic conditions (e.g., various traffic parameters such as vehicular locations, velocities, and directions). As presented in, the running time of the optimal solution increases sharply with an increasing number of requestors and helpers. The optimal running time is 88.83 seconds, whereas that of the proposed mechanism is only around. 


\section{EPRA International Journal of Research and Development (IJRD)}

Volume: 6 | Issue: 9 | September 2021

- Peer Reviewed Journal

\section{SAMPLE SCREENSHOTS}

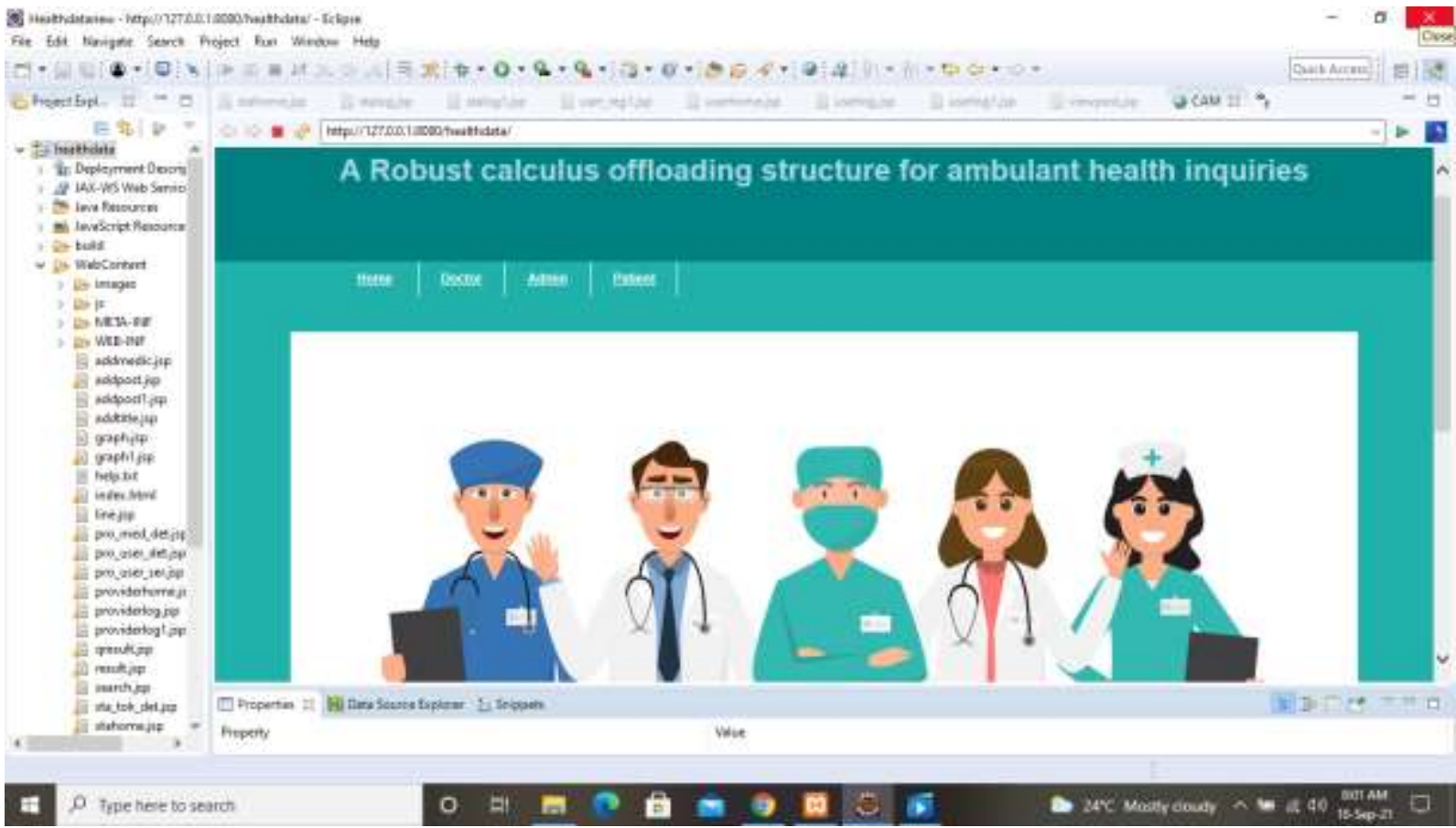

FIG 2. This is the home screen for this application.

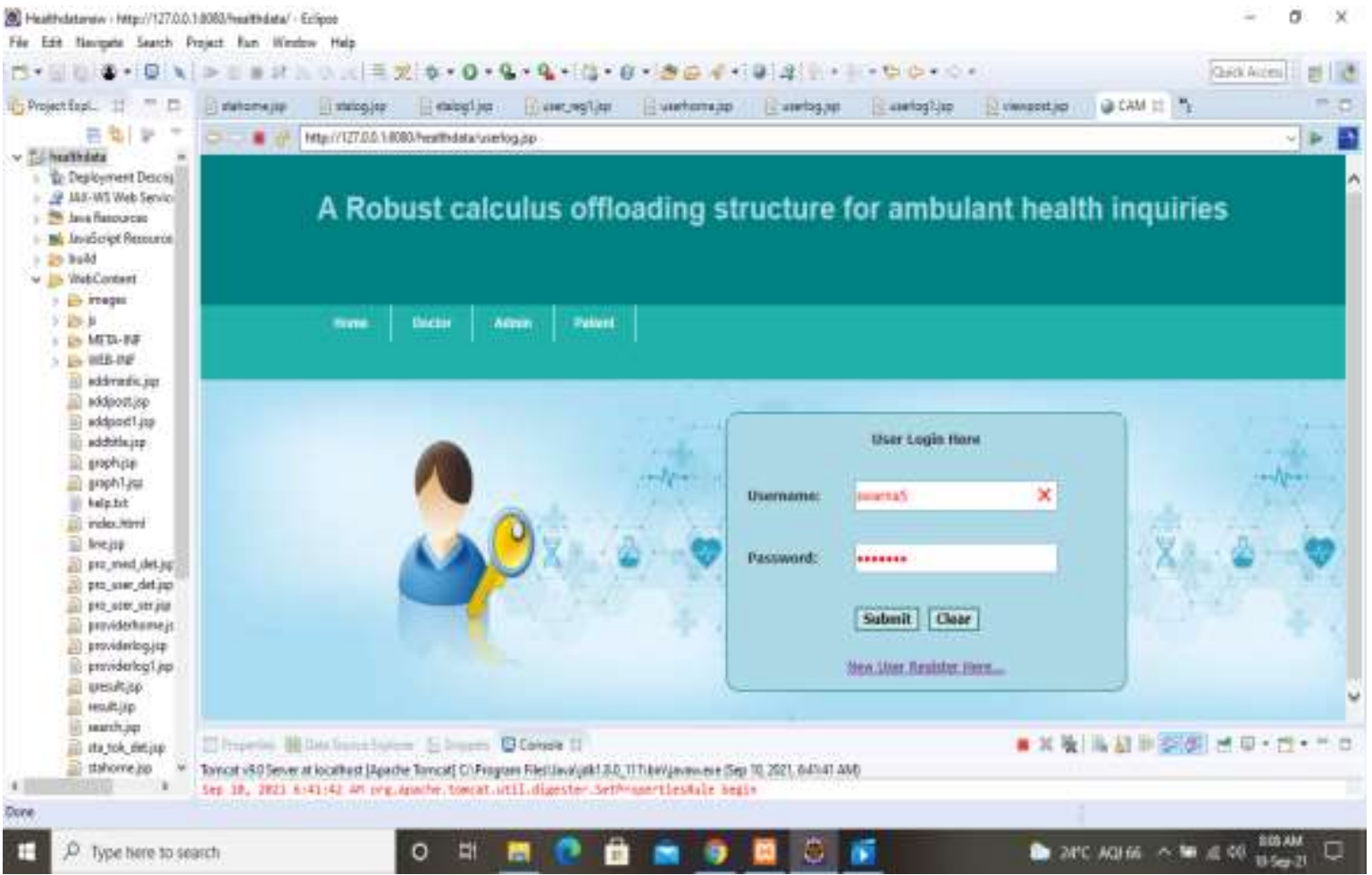

FIG 3. Patient can register and Login here with their details. 


\section{EPRA International Journal of Research and Development (IJRD)}

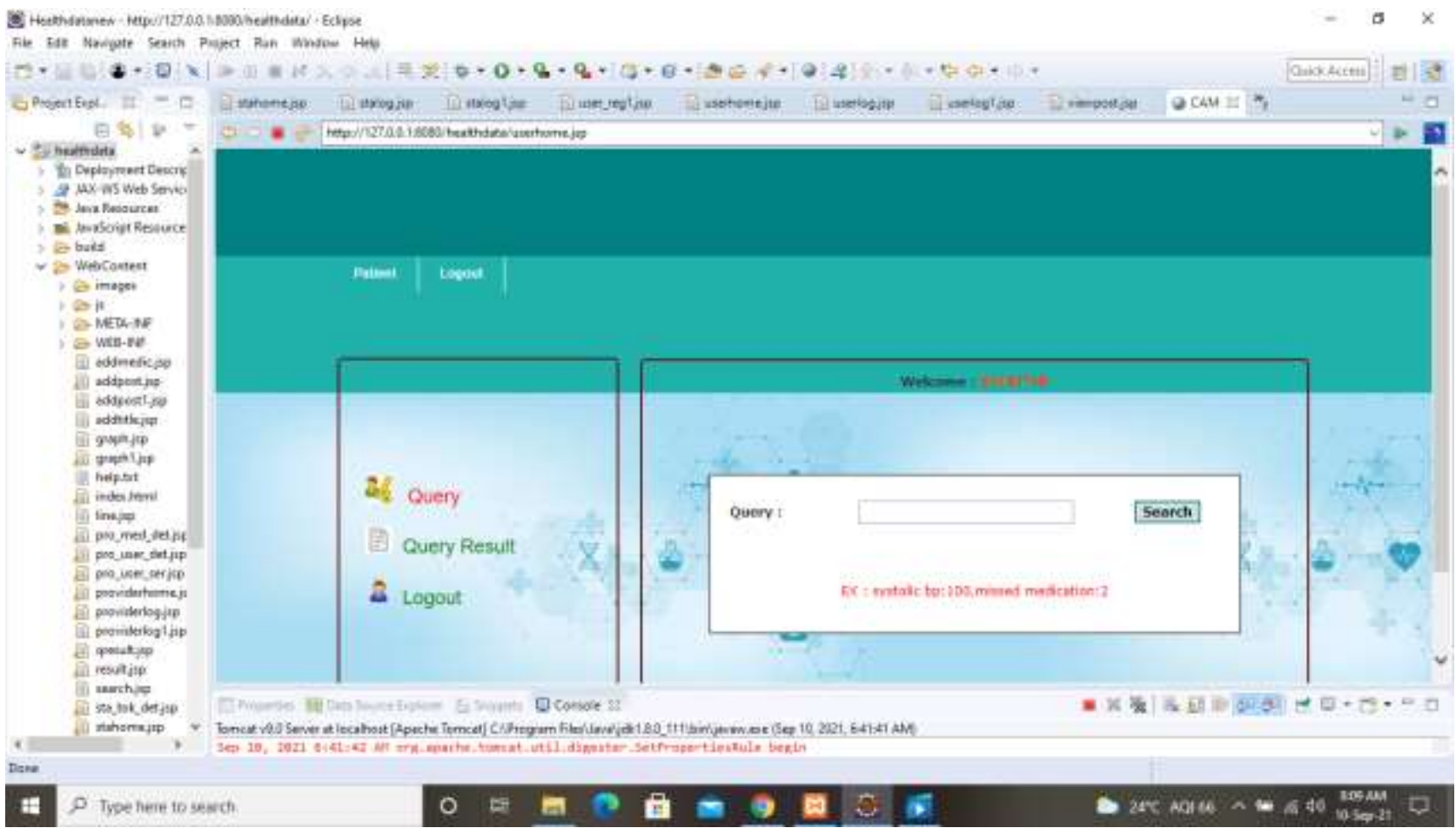

FIG 4. Patient Register specific concern.

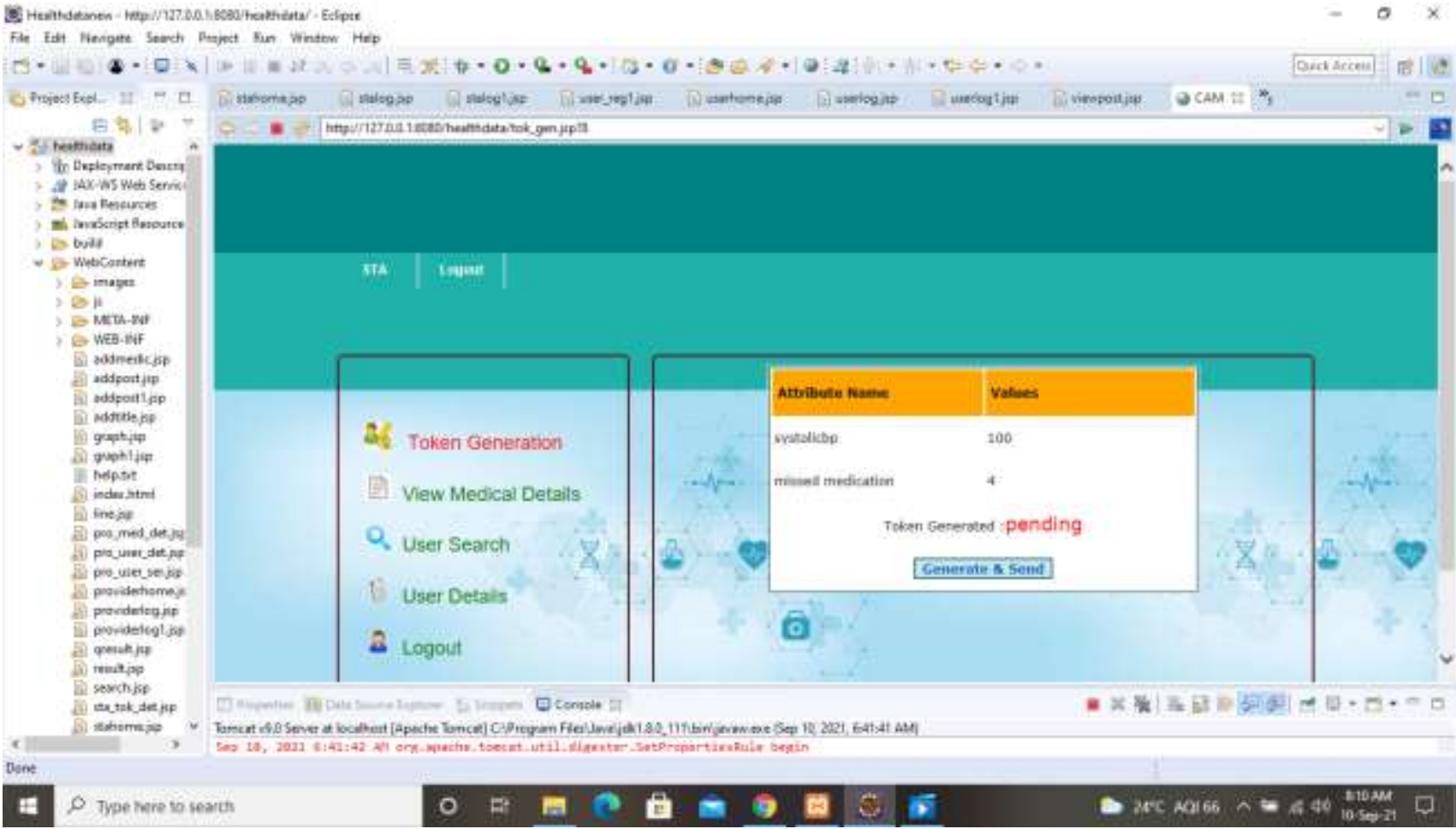

FIG 5. Token will be Generated to Doctor. 


\section{EPRA International Journal of Research and Development (IJRD)}

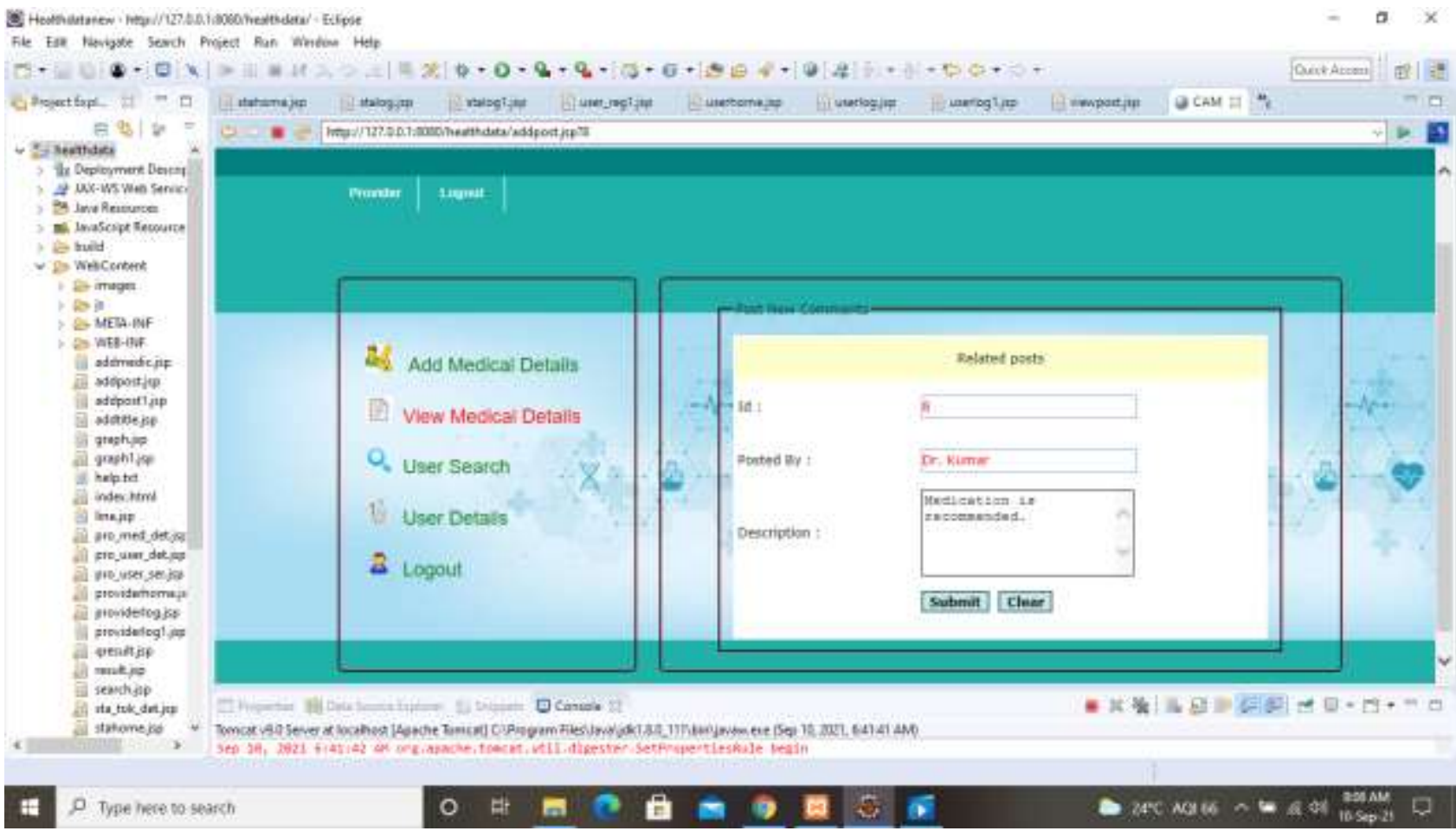

FIG 6. Doctor views the concern and respond to it.

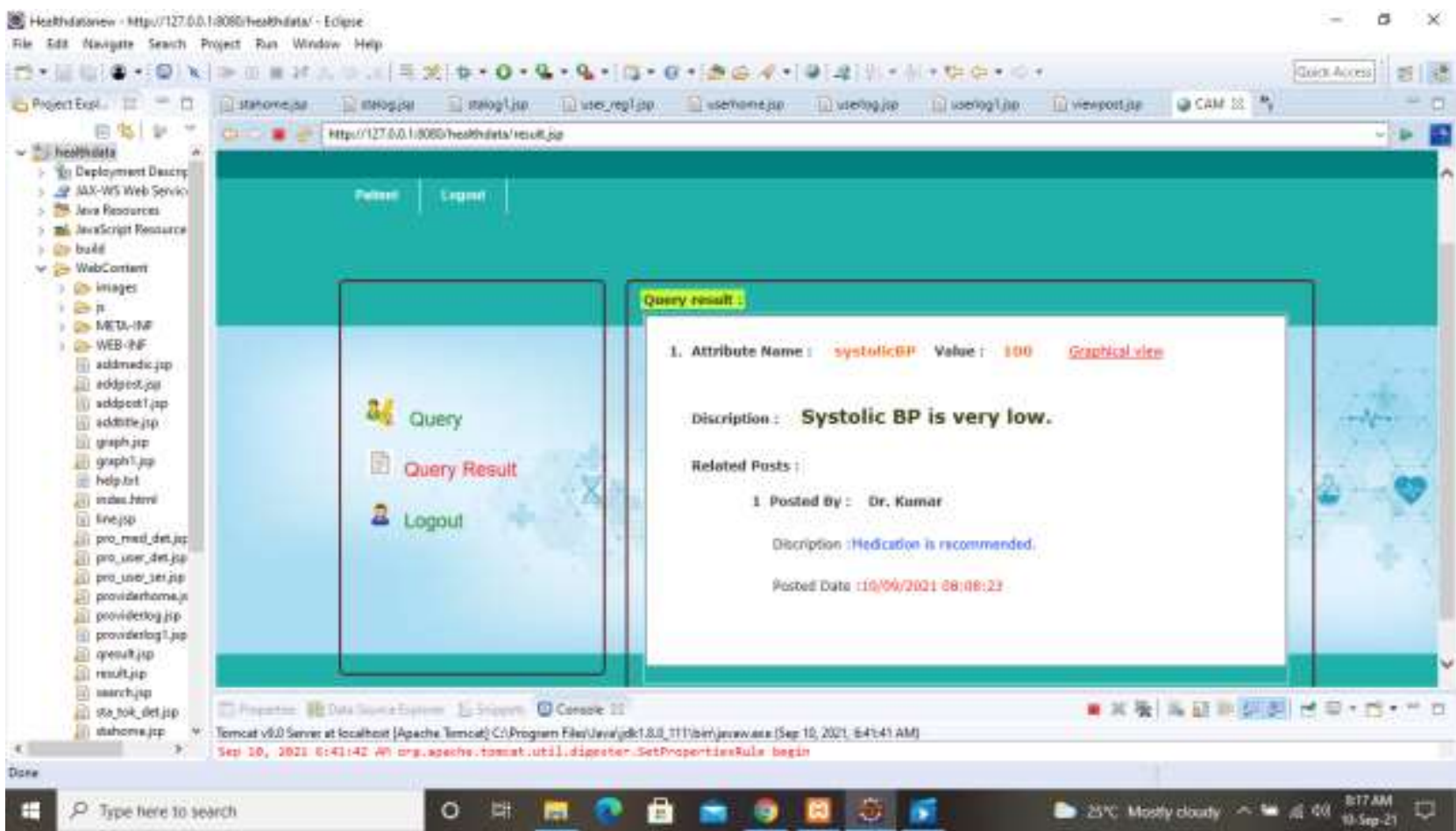

FIG 7. Patient login and views the result. 


\section{EPRA International Journal of Research and Development (IJRD)}

\section{CONCLUSION}

This paper presents a computation offloading mechanism for mobile health applications based on $\mathrm{CAD}$ in medical disaster scenarios, where a selforganizing network of vehicles is applied. With the advantages of edge computing and $\mathrm{V} 2 \mathrm{~V}$ communication technologies, rescue organization can efficiently solve computation offloading problems for mobile health applications. We propose a novel computation offloading mechanism by formulating a non-linear integer programming problem, where each application is divided into several parts and offloaded to nearby vehicles. As the problem is NP-hard with exponentially increasing computational complexity, a PSO algorithm is proposed for scenarios with denser vehicles.

\section{REFERENCES}

1. R. S. Ledley and L. B Lusted, "Reasoning foundations of medical diagnosis; symbolic logic, probability, and value theory aid our understanding of how physicians reason," Science, vol. 130, no. 3366, pp. 9-21, 1959.

2. $\quad R$. Eberhart and J. Kennedy, "A new optimizer using particle swarm theory," in Proc. 6th Int. Symp. Micro Mach. Hum. Sci. 1995, pp. 39-43.

3. T.Case, C. Morrison, and A.Vuylsteke, "The clinical application of mobile technology to disaster medicine," PrehospitalDisaster Med., vol. 27, no. 5, pp. 473-480, 2012.

4. H.Magsi, A. H. Sodhro, and F. A. Chachar, "Evolution of $5 G$ in Internet of Medical Things," in Proc. Int. Conf. Comput., Math. Eng. Technol., 2018, pp. 1-7.

5. A. H. Sodhro, F. K. Shaikh, and S. Pirbhulal, "Medical-QoS based telemedicine service selection using analytic hierarchy process," Handbook of Large-Scale Distributed Computing in Smart Healthcare, 2017, pp. 589-609.

6. S. Tachakra, X. H. Wang, R. S. H. Istepanian, and Y. H. Song, "Mobile e-health: The unwired evolution of telemedicine," Telemed. J. e-Health vol. 9, no. 3, pp. 247-57, 2003.

7. E. Kartsakli, A. S. Lalos, A. Antonopoulos, S. Tennina, M. D. Renzo, and L. Alonso, "A survey on M2M systems for mHealth: A wireless communications perspective," Sensors, vol. 14, no. 10, pp. 1800918052, 2014.

8. H. T. Dinh, C. Lee, D. Niyato, and P. Wang, "A survey of mobile cloud computing: Architecture, applications, and approaches, "Wireless Commun. Mobile Comput., vol. 13, no. 18, pp. 1587-1611, 2013.

9. G. R. Tiago, S. Katsuya, N. Hiroki, and K. Nei, "Hybrid method for minimizing service delay in edge cloud computing throughVM migration and transmission power control," IEEE Trans. Comput., vol. 66, no. 5, pp. 810-819, May 2017.

10. G. R. Tiago, S. Katsuya, N. Hiroki, and K. Nei, and K. Temma, "Cloudlets activation scheme for scalable mobile edge computing with transmission power control and virtual machine migration," IEEE Trans. Comput., vol. 67, no. 9, pp. 1287-1300, Sep. 2018. 